\title{
Research on Multi-agent System Automated Negotiation Theory and Model
}

\author{
Weijin Jiang ${ }^{1}$, Yusheng $\mathrm{Xu}^{2}$, Ding $\mathrm{Hao}^{1}$, and Shangyou Zhen ${ }^{2}$ \\ ${ }^{1}$ Department of computer, Zhuzhou Institute of Technology, Zhuzhou 412008, P.R. China \\ jwjnudt@163. com \\ ${ }^{2}$ College of Mechanical Engineering and Applied Electronics, \\ Beijing University of Technology, Beijing 100022, P.R. China \\ yshxu520@163.com
}

\begin{abstract}
The communication between agents has some special requirements. One of them is asynchronous communication. Used communication sequence process (CSP) to descript a model of agents communication with shared buffer channel. The essence of this model is very suitable for the multi-agents communication, so it is a base for our next step job. Based on the communication model, explored the distributed tasks dealing method among joint intention agents and with description of relation between tasks we give a figure of agents' organization. Agents communicate with each other in this kind of organization. The semantics of agent communication is another emphasis in this paper. With the detailed description of agents' communication process, given a general agent automated negotiation protocol based on speech act theory in MAS, then we use CSP to verify this protocol has properties of safety and liveness, so prove it is logic right. At last a frame of this protocol's realization was given.
\end{abstract}

\section{Introduction}

The theory of Multi-Agent Automated Negotiation involves extensive applying fields and many kinds of methods. The theory mainly lies in Argument Based Automated Negotiation, Game Theoretic Models and Heuristic Approaches. In application, it can be divided into two categories, Agent's Negotiation within MAS and Self-interested between different MAS. Those theories supporting the interior collaboration of MAS are like Self-interested, Joint Intentions and Shared Plans, no matter which are have differences, they have been working under the premise of identical intention and target of Agent within MAS. This text will discuss the Joint Intentions in Multi-Agent Automated Negotiation of MAS[1-4].

If Multi-Agent in MAS interacts successfully, there must be three conditions demanded to be satisfied as below:

1) Communication Structure, that is, how to dispatch and take over information between Agent ;

2) Communication Language, that is, Agent is required to understand the signification of the information;

3) Interaction Rules, that is, how to organize the conversation between Agent. 
Regarding to the research of Agent Communication Structure, we have proposed TTMAS communication model in the previous parts. In the second section, it will be stressed to analyze Agent's asynchronous communication mechanism[5,6]. As to the research of Agent Communication Language, presently there have been many abroad, like KQML, FIPA, ACL, Agent Talk, etc., so the language is not the emphasis in our text. Then, research of Interaction Rules is the second emphasis in the text. In the third part, the text will set forth the agreement of Agent Automated Negotiation and its validation. In the forth part, it illustrates and analyzes the complete frame of Agent Automated Negotiation. The fifth is the conclusion of the text.

\section{Agent Communication Mechanism Analyses}

Definition 1. Agent is a status course which can accomplish the task automatically with the ability and agreement of communication, for example, $P_{\mathrm{A}}$ represents the course of Agent A.

Definition 2. The course of Agent make the Agent's ability which can be marked as Ability $_{P_{A}}$ and TASK $_{P_{A}}$ means to be able to fulfill the task.

The moving status of the static Agent in MAS can be classified as Active, Wait and Run. Agent in the Wait status will be activated after receiving the requests from other Agent and then run. Agent in Run status will negotiate with other Agent or provide

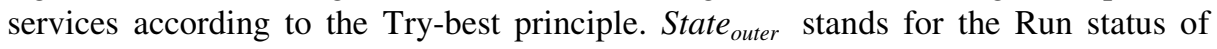
Agent:

State $_{\text {outer }}::=$ Wait | Active | Run

Agent's collaborating course observed from the outer MAS is the process that Agent runs in the $\mathrm{I}_{\text {outer }}=$ State $_{\text {outer }}{ }^{*}$

Definition 3. Contain the protocol system extremely locking the state, including STOP process in its CSP expression formula.

Definition 4. Contain alive protocol system that lock, its CSP expression formula will certainly include part exported to have pass ring of returning.

Theorem 1. In an Agent's collaborating process with Safety and Liveness, the circulation of Wait $\rightarrow$ Active $\rightarrow$ Run $\rightarrow$ Wait in $\mathrm{I}_{\text {outer }}$ will appear at least once to Agent's launch and acceptance.

Attestation : Obviously, in the circulation of Wait $\rightarrow$ Active $\rightarrow$ Run $\rightarrow$ Wait, if any one part of Agent can not fulfill the circulation, it means something happened unexpectedly cause the deadlock or livelock to the system during the collaborating process, so the theorem attested.

Definition 5. Buffer channel $C$ is such an Agent which set independent state switch and message buffer to all its relevant Agents and transmit messages for these Agents. 


\section{MAS Interior Agent Cooperation Model}

When Multi-Agent in MAS begins cooperation, for the reason that there is a conform joint intension between Agent, the process of Multi-Agent in MAS works according to the principal of "From each according to his ability, abide by the law and behave oneself", that is, each Agent is trying its best to cooperated with other Agent[7,8].

\subsection{Automatic Negotiation in Agent Protocol}

Agent automatic negotiation is the main method for multi-Agent to negotiate, which focus on three aspects lieing in negotiation protocol, negotiation object and negotiation policy. Negotiation protocol and negotiation object act as the textual points, but the negotiation policy is clampinged how to look for in Agent each from of negotiation space best in order to reach consistence, concretion content visible literature cited.

Present hypotheses 1 to ensure negotiation agent could each other have partner faith in against due to MAS interior Agent according to Try-Best principle proceed synergic, furthermore MAS possess concurrent combine intent.

Hypotheses 1. Negotiation Agent knows each other in negotiation policy.

$\mathrm{Be}$ on the negotiation with the result that decision agent toward inter network communication negotiatory condition of Agent automatic negotiatory course mission due to specific assignment require different communication quality guarantee AND specific network insurance. Text take mission negotiation AND inter network communication negotiation as agent automatism negotiation in process two phase.

Definition 6. MAS interior agent automatic negotiation course could include two phases. The first phase is based on multi-Agent automatic negotiation whose negotiation object includes task starting time, task ending time and the relation of the tasks; The second phase is the negotiation of Agent's communicating conditions whose negotiation object include corresponding security policy and network service quality $\left(\mathrm{Q}_{\mathrm{O}} \mathrm{S}\right)$.

According to the top analysis talks about with the correlative language behavior academic theories, we say the Agent automatic negotiation correspondence in the procedure to state row word certain for: request, promise, refuse, advise, counter advise. In view of agreement presence overtime event and agent unsolicited message transmission, so increase overtime (timeout) status and inform (inform) state row word that. Communication protocol engine of the communication process state as follows of the agent :

State $_{\text {inner }}::=$ Started 1 Requested | Accepted | Refused | Promised | Informed | Advised | CAd-vised | Timeout | Stopped

Agent automatic negotiation protocol can be divided into information transmission layer, buffer channel layer and Agent negotiation protocol layer from bottom to top, of which buffer channel layer $\mathrm{C}$ is one of the needed layers between Agents to realize asynchronous communication. If it will realize point-to-point synchronous 
communication between Agents, it can do communication directly through channel C. As to the description of Agent automatic negotiation, it mostly focus on Agent negotiation protocol layer, while for the other layers, it only describes their services and running environment in brief. In essence, the function of Agent negotiation protocol layer is the description of process.

The service provided by each protocol layer:

a. Information transmission layer: being in position to transmit information data between Agents in sequential way and correctly;

b. Buffering channel $\mathrm{C} 0$ and $\mathrm{C} 1$ layer: providing Agent automatic negotiation layer with the services described in 2.2.;

c. Agent automatic negotiation protocol layer : supplying Agent with credibility, efficient negotiation control and policy.

\section{Conclusions}

This text provides a common and communication-based Agent cooperation mode by studying mutual behavior of Agent cooperation. The text also uses some effective format ways to depict automatic negotiation protocol of Agent process and verify the validity of the protocol's logic. Finally, the text makes an implementation frame for this agreement. While using blackboard mode to realize buffer channel in this implementation frame, it provides a deployed agreement stack extra and at last it presents performance analysis and expandable analysis. In addition, as to negotiation between Agent in MAS, because the advantage difference of Agent group negotiating with Agent which has a conform joint intension has great differences on negotiation principle and strategy, the self-interested Agent's negotiation agreement between MAS is our next work under research.

\section{References}

1. Jennings $\mathrm{N}$ R ,Faratin P, Lomuscio A R et al.: Automated negotiation: prospects. Methods and challenges[C]. Pacific Rim International Conference on Artificial Intelligence, (2000)

2. Grosz B, Sidner C.: Plans for discourse[A]. In: P. Cohen, Morgan J, Pollack M. eds. Intentions in communication [M]. Bradford Books, MIT Press, (1990)

3. Wang Bin . Zhang Yao-xue, Chen Song-qiao: A communication method of MAS based on blackboard architecture[J]. Mini-Micro Systems, 23(11), (2002) 1355-1358

4. In G. Agha and F.: Decindio, editors, Concur-rent Object-Oriented Programming and Petri Nets, Lecture notes in Computer Science[M]. Springer-Verlag, Berlin, (1998)

5. Jiao Wen-pin, Shi Zhong-Zhi.: Modeling dynamic architectures for multi-agent system[J]. Chinese Journal of Computers, 23(7), (2000) 732-737

6. Mao Xin-jun: Anon-terminating active computing model in multi-agent systems[J]. Journal of Computer Research \& Development, 36(7) , (1999) 769-775

7. Jiang Weijin: Modeling and Application of Complex Diagnosis Distributed Intelligence Based on MAS. Journal of Nanjing University(Natural Science), 40(4), (2004) 483-496

8. Jiang Weijin: Research on Diagnosis Model Distributed Intelligence and Key Technique Based on MAS. Journal of Control Theory \& Applications, 20(6), (2004) 231-236 